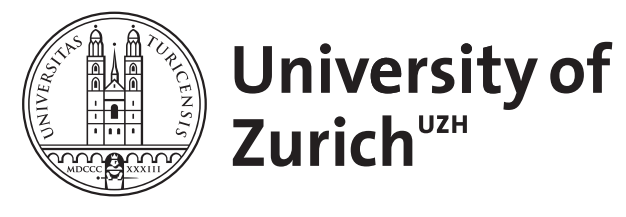

\title{
Who matters in coordination problems?
}

Sákovics, József ; Steiner, Jakub

\begin{abstract}
Agents face a coordination problem akin to the adoption of a network technology. A principal announces investment subsidies that, at minimal cost, attain a given likelihood of successful coordination. Optimal subsidies target agents who impose high externalities on others and on whom others impose low externalities. Based on the analysis of the role of strategic uncertainty in coordination processes, we provide a methodology that can be used to find the optimal targets for a variety of interventions in a large class of coordination problems with heterogeneous agents.
\end{abstract}

DOI: https://doi.org/10.1257/aer.102.7.3439

Posted at the Zurich Open Repository and Archive, University of Zurich

ZORA URL: https://doi.org/10.5167/uzh-174740

Journal Article

Published Version

Originally published at:

Sákovics, József; Steiner, Jakub (2012). Who matters in coordination problems? American Economic Review, 102(7):3439-3461.

DOI: https://doi.org/10.1257/aer.102.7.3439 


\title{
Who Matters in Coordination Problems? ${ }^{\dagger}$
}

\author{
By József SÁkovics and JaKub SteineR*
}

\begin{abstract}
Agents face a coordination problem akin to the adoption of a network technology. A principal announces investment subsidies that, at minimal cost, attain a given likelihood of successful coordination. Optimal subsidies target agents who impose high externalities on others and on whom others impose low externalities. Based on the analysis of the role of strategic uncertainty in coordination processes, we provide a methodology that can be used to find the optimal targets for a variety of interventions in a large class of coordination problems with heterogeneous agents. (JEL D81, D82, D83, O33)
\end{abstract}

Coordination failures can have enormous social costs. Perhaps the most important examples are missed opportunities for economic development, but coordination problems also arise in many other contexts. ${ }^{1}$ The risk of a damaging coordination failure invites intervention. As intervention is costly, the policymaker will want to target the agents who have the largest overall impact on the coordination process.

Turning to the current economic crisis, which agents are most influential in the process of recovery? Those producing tradable or nontradable goods? Those in procyclical or in counter-cyclical sectors? International or domestic investors? All these groups differ in many dimensions, including their access to capital and their benefits from high overall economic activity. Should policies be targeted at those with large or low benefits? At those with low or high investment costs? Distinguishing the influential agents within the coordination process is a complex task, as in the presence of externalities the incentives of any single group affect the equilibrium behavior of all the other agents as well. Thus, without a formal model, it is hard to identify the optimal target for intervention.

\footnotetext{
*Sákovics: The University of Edinburgh, School of Economics, 31 Buccleuch Place, Edinburgh, EH8 9JT, United Kingdom (e-mail: jozsef.sakovics@ed.ac.uk); Steiner: CERGE-EI, joint workplace of Charles University Prague and the Economics Institute of the Academy of Sciences of the Czech Republic, Politických vězňu 7, Praha, Czech Republic and Northwestern University (e-mail: j-steiner@kellogg.northwestern.edu). We are grateful to the anonymous referees for their constructive criticism. We also thank Tijmen Daniëls, Gianni De Fraja, Itay Goldstein, Frank Heinemann, Sergei Izmalkov, Eugen Kováč, Laurent Mathevet, John Moore, Stephen Morris, Tymofiy Mylovanov, Alessandro Pavan, Nicola Persico, Rani Spiegler, Colin Stewart, Yosuke Yasuda, seminar participants at UT-Austin, UBC, Cambridge, CERGE, CEU, UC-Davis, U. Edinburgh, Humboldt, Penn State, Queens (Belfast), Stanford, and TU Berlin, and conference participants at EEA-ESEM 09, and CETC 10 for helpful comments. The second author has benefited from ESRC grant RES-061-25-0171, and from Purkyne Fellowship of the Academy of Sciences of the Czech Republic

${ }^{\dagger}$ To view additional materials, visit the article page at http://dx.doi.org/10.1257/aer.102.7.3439.

${ }^{1}$ Indeed, the founding fathers of development economics thought of the lack of economic development as a massive coordination failure (Ray 2000). Additional applications include bank runs (Diamond and Dybvig 1983), standard setting (Farrell and Saloner 1985), technology adoption (Katz and Shapiro 1986), the use of fiat money (Kiyotaki and Wright 1989), law enforcement (Sah 1991), and currency attacks (Obstfeld 1996), just to name a few.
} 
We derive novel results for large coordination games with heterogeneous agents that allow us to give policy advice in a wide range of situations. In the canonical case of investment subsidization, we find that ideal candidates for the subsidy need to satisfy only two criteria: (i) their investment has a relatively large direct impact on the incentives of others, but (ii) they are relatively insensitive to the investment of others.

Consider the example of how shopping mall rental fees are set. Mall developers, unlike office building developers, use subsidies (rent discounts) to coordinate the shops on locating in their mall. Vindicating both our criteria, Pashigian and Gould (1998) document that "anchor" shops-typically, brand name department storesreceive large rent discounts. Anchor shops bring in loyal customers who end up shopping at other stores as well; thus, their decisions have a large impact on the decisions of others. At the same time, their sales are relatively unaffected by the custom of shoppers derived from other stores, as shown by the similarity of their sales per square foot between regional and superregional malls. ${ }^{2}$

Beyond the example of direct subsidies, our approach is useful for targeting any type of intervention aimed at coordinating heterogeneous groups. For example, carefully describing the different payoff structures of domestic and foreign depositors, König and Daniëls (2011) employ our methodology to show how to strike the right balance between holding international reserves and setting a term-interest structure on debt contracts in order to decrease the likelihood of coordination on the bank-run equilibrium in emerging markets. We discuss further applications, including network goods and financial fragility, in Section VI, following the presentation of our results.

To illustrate the complexities of coordination problems, let us examine a stripped down, two-person version of our model. Two agents decide simultaneously whether to invest into a common risky project. The project succeeds if they both invest and fails otherwise. Not investing pays 0 , investing costs $c_{i}$ and leads to a benefit $b_{i}>c_{i}$, but only if the project succeeds. The agents face a coordination problem as mutual investing or not investing both constitute an equilibrium.

Consider a policymaker who, in order to encourage coordination on investing, reduces the investment costs to $\tilde{c}_{i}<c_{i}$ by publicly offering subsidies, $s_{i}=c_{i}-\tilde{c}_{i}$, to investing players. To ensure that mutual investment becomes the unique equilibrium, she would have to make investing dominant for only one of the agents (the other agent would respond by voluntary investment). Therefore, the cost of such a policy would be the lower of the two investment costs. Often, this cost may be too high; a policymaker may lack the resources for such a "big push." 3 To be effective, such a weak policymaker needs to understand the finer mechanics of how the agents coordinate their actions. In particular, she needs to decipher how they form their beliefs about the actions of others-their strategic beliefs.

\footnotetext{
${ }^{2}$ Gould, Pashigian, and Prendergast (2005) delve further into the details of mall rental contracts and document that the overage sharing percentage of anchors is also significantly lower than that of the other stores. This observation shows that the coordination game is played out not only at the location level but also at the continuing effort level. As it is important for the mall that their loyal customers keep coming, it has to incentivize the department stores to put in an amount of effort which is commensurate with the externalities they generate. Our insights for subsidy targeting apply here equally.

${ }^{3}$ The budgets of even the largest financial institutions, such as the International Monetary Fund (IMF), are small compared to the volume of investment they hope to influence. The IMF thus can only engage in small, "catalytic finance" interventions, and the success of these depends on their impact on the beliefs of private investors. See Corsetti, Guimarães, and Roubini (2006), and Morris and Shin (2006).
} 
To get a feeling for the complex effects of small subsidies, take the scheme that subsidizes Agent 1 only. While a small subsidy does not make investing dominant for her, it makes her more likely to invest. Indirectly, it makes Agent 2 more eager to invest as well, as he becomes more optimistic about Agent 1's investment. This further increases Agent 1's incentives to invest, etc. Though both equilibria-and, hence, strategic uncertainty-persist, intuitively, the chance that the agents coordinate on investment increases. But which agent should get the subsidy?

The impact of strategic uncertainty on the agents' behavior is well captured by the risk dominance criterion. Roughly speaking, the investing equilibrium is risk dominant if rationalizing investment does not require too much optimism from the players. ${ }^{4}$

To introduce risk dominance in our example, define $p_{i}^{*}=\tilde{c}_{i} / b_{i}$ as the minimal probability Agent $i$ needs to assign to her partner investing, so that she herself is willing to invest. Interpreting $1-p_{i}^{*}$ as a measure of Agent $i$ 's predisposition to invest (and $p_{i}^{*}$ of not to), the average of the predispositions to invest has to exceed the average of the predispositions not to invest for coordination on investment to happen. Formally, mutual investment is the (weakly) risk dominant equilibrium if $p_{1}^{*}+p_{2}^{*} \leq 1$.

When the agents follow the risk dominance criterion, the policymaker's problem is to minimize subsidy expenditures, $c_{1}+c_{2}-\tilde{c}_{1}-\tilde{c}_{2}$, subject to

$$
p_{1}^{*}\left(\tilde{c}_{1}\right)+p_{2}^{*}\left(\tilde{c}_{2}\right) \leq 1
$$

\section{We call inequality (1) the belief constraint.}

The unique optimal policy is simple and sensible. The subsidy is targeted exclusively at the agent who cares less about the success of the project: the one with the lower benefit parameter. ${ }^{5}$ This scheme is intuitively attractive even without appealing to the risk dominance criterion. It directly motivates the agent who is relatively insensitive to her partner's action (and hence is more difficult to motivate indirectly), while her partner is motivated indirectly, by exploiting his sensitivity to the subsidized agent's action.

In our main model we subscribe to the "global game" justification of the risk dominance criterion in Carlsson and van Damme (1993). The heart of their argument is that a small private noise in the observation of payoffs may lead to large strategic uncertainty. Assume that the two agents receive correlated private real-valued signals, $x_{i}$, and they invest if and only if their signals exceed their respective thresholds, $x_{i}^{*}$. The authors observe that, in the absence of prior information, the probabilities that the players who receive the threshold signals assign to their partner investing sum to one: ${ }^{6}$

$$
\operatorname{Pr}\left(x_{2}>x_{2}^{*} \mid x_{1}=x_{1}^{*}\right)+\operatorname{Pr}\left(x_{1}>x_{1}^{*} \mid x_{2}=x_{2}^{*}\right)=1
$$

\footnotetext{
${ }^{4}$ The criterion was proposed by Harsanyi and Selten (1988), and was later derived from evolutionary arguments by Kandori, Mailath, and Rob (1993), from infection arguments due to private information by Carlsson and van Damme (1993), and from stability arguments in the perfect foresight model of Matsui and Matsuyama (1995). For ample experimental evidence that people tend to coordinate on risk dominant equilibria, see Camerer (2003).

${ }^{5}$ Suppose $b_{1}<b_{2}$. The necessary subsidy $s_{1}$ solves $\left(c_{1}-s_{1}\right) / b_{1}+c_{2} / b_{2}=1$. It is strictly less than the one needed for full implementation, which — as we have seen above-would be the lower of the two cost parameters.

${ }^{6}$ The argument behind equation (2) is simple. With uninformative priors, the posteriors can be rewritten as $\operatorname{Pr}\left(x_{2}>x_{2}^{*} \mid x_{1}=x_{1}^{*}\right)=\operatorname{Pr}\left(x_{2}-x_{1}>x_{2}^{*}-x_{1}^{*}\right)$ and $\operatorname{Pr}\left(x_{1}>x_{1}^{*} \mid x_{2}=x_{2}^{*}\right)=\operatorname{Pr}\left(x_{2}-x_{1}<x_{2}^{*}-x_{1}^{*}\right)$. These two terms add to one for any atomless distribution of $x_{2}-x_{1}$.
} 
In words, an "opinion poll" of the two threshold types reveals no information-it assigns probability $1 / 2$ to each of the two possible partner's actions. It turns out that, in a natural setup, this constraint on the threshold types' strategic beliefs implies that the agents choose the risk-dominant actions.

Most economically relevant coordination problems involve many players. Thus, we consider a continuum of heterogeneous agents (making the standard risk dominance criterion not applicable). Success arises if the aggregate investment exceeds a critical mass. We generalize the risk dominance criterion: agents coordinate on investing if the success of the project does not require too large participation compared to the average predisposition to invest. The policy recommendation generalizes as well: the expenditure minimizing planner should target subsidies at the agents (i) whose actions have large effect on the aggregate action, but (ii) whose own incentives are relatively insensitive to variations in the aggregate action.

We obtain this result in a global game setup in which the critical mass is uncertain but each player receives a nearly perfect private signal about it. The game has a unique equilibrium in which all players follow threshold strategies. Subsidies shift the equilibrium threshold signals toward more demanding projects and hence they increase the probability of coordination on investing.

As in the $2 \times 2$ setup of Carlsson and van Damme, we focus on the strategic beliefs of the threshold types. While these beliefs are quite complex, they turn out to satisfy a simple identity. We find that the average of the threshold types' strategic beliefs - captured as probability distributions over the aggregate action-is the uniform distribution. As before, an "opinion poll" among the threshold types reveals no information about the aggregate action.

The last property is a generalization of the belief constraints (1) and (2) for the interaction of many players. It holds independent of the error distributions or investment incentives. Thanks to its robustness, the property holds in a large class of setups and allows us to characterize the coordination outcome and specify the optimal policy for a broad range of payoffs and information structures.

The paper is organized as follows. After reviewing the related literature, we describe our model in Section II. Section III characterizes the unique equilibrium in the limit of precise signals, and also discusses our main technical result: the belief constraint. In Section IV, we apply the characterization result to derive the optimal subsidy schemes. Section V discusses the intuition and implications of the optimal policies. Section VI presents applications, together with alternative interventions. Section VII concludes. In the main text we work with the simplest possible payoff functions; the proofs in the online Appendix assume richer payoff structures, allowing for a larger modeling flexibility.

\section{Related Literature}

Compared to the Pigouvian planner, our rather weak and uninformed planner is less ambitious. Instead of trying to achieve the first best, she only aims to increase the incidence of investment. After all, she tackles a harder problem as she has to deal with the strategic uncertainty that our agents face. Within the contracting literature, the seminal papers of Segal $(1999,2003)$ capture the planner's uncertainty about 
the coordination outcome, but still assume that the agents themselves do not face strategic uncertainty in equilibrium.

Bernstein and Winter (2012) study subsidies inducing efficient coordination in a complete information setting. Like Segal (2003) and unlike us, they focus on full implementation. They show that the optimal policy is to divide and conquer: a judiciously chosen subset of players is subsidized, so that they invest even if no one in the complement set invests. The rest of the players will then invest even if they are taxed to the level of indifference, as they can take for granted the investment of the subsidized players. Returning to our two-player example: $i$, who receives the subsidy $c_{i}$, invests even if he assigns probability $p_{i}^{*}=0$ to $j$ investing, whereas $j$, who is taxed, invests only if she assigns probability $p_{j}^{*}=1$ to $i$ investing. Thus, as in our case, the planner ends up choosing schemes that satisfy the belief constraint $p_{1}^{*}+p_{2}^{*}=1$, but she considers only corner solutions; $\left(p_{1}^{*}, p_{2}^{*}\right)=(0,1)$ or $(1,0)$. In contrast, our planner is constrained to choose schemes from a subset of the interior of the belief constraint.

As stated before, our paper belongs to the global game literature originated by Carlsson and van Damme (1993). The most commonly applied global game setup, reviewed in Morris and Shin (2003), consists of a symmetric game with strategic complementarities played by many players who share a common payoff function. ${ }^{7}$ In the unique equilibrium, every player follows the same threshold strategy. This greatly simplifies the analysis as the threshold type turns out to have no information about the aggregate action: she believes that the aggregate action is distributed uniformly. This property is a special case of our belief constraint when applied to a homogeneous population.

The analysis of global games with heterogeneous payoffs is harder. Frankel, Morris, and Pauzner (2003) prove equilibrium uniqueness for a large class of these games. Explicit solutions are only known for a few particular setups: ${ }^{8}$ Morris and Shin (2003) note that heterogeneity in the quality of private signals has no consequence in a two-action global game in the absence of payoff heterogeneity. Corsetti et al. (2004) characterize the impact of a large trader on a population of small ones. Guimarães and Morris (2007) allow for payoff heterogeneity in a model of currency attacks and find that risk-averse speculators have a surprisingly high influence on the attack. As risk aversion decreases the utility benefit from a successful attack, this finding conforms to our result. ${ }^{9}$

Two recent papers study the impact of heterogenous strategic beliefs on coordination processes. Izmalkov and Yildiz (2010) assume exogenous differences in the prior beliefs, while Mathevet (2011) focuses on the comparison of strategic beliefs across multiple equilibria. The heterogeneity of strategic beliefs arises in a unique equilibrium and under common priors in our model. It is an endogenous equilibrium phenomenon, driven by the heterogeneity of incentives.

\footnotetext{
${ }^{7}$ It has been used to study currency attacks (Morris and Shin 1998), bankruptcies (Morris and Shin 2004a), liquidity crises (Morris and Shin 2004b), bank runs (Goldstein and Pauzner 2005), political revolutions (Edmond 2011), and other coordination problems.

${ }^{8}$ Some applied papers studying heterogeneous global games avoid the explicit solution and focus on comparative statics instead. See, for example, Goldstein and Pauzner (2004).

${ }^{9}$ Guimarães and Morris (2007) characterize equilibrium using a different technique from ours. Their proof exploits the simplicity of the binary payoff functions but incorporates continuous investment levels. Our proof, based on the belief constraint, relies on a binary action space, but applies to a large set of payoffs.
} 
Finally, Bebchuk and Goldstein (2011) study bailouts in a model that complements ours. While we focus on the heterogeneity of incentives and hence on the targeting of interventions, they assume homogeneous incentives and focus on sophisticated schemes contingent on the performance of the economy.

\section{The Model}

A continuum of players of measure $m$, indexed by $i \in[0, m]$, make simultaneous binary investment decisions, $a_{i} \in\{0,1\}$, into a common project that either succeeds or fails. The players are heterogeneous both in their investment incentives and in their influence over the aggregate action. Each player $i$ is characterized by three parameters: weight $w_{i}>0$; cost of investment $c_{i}>0$; and benefit from the success of the project $b_{i}>c_{i}$. The first parameter determines each player's influence over the aggregate action, $a=\int_{0}^{m} w_{i} a_{i} d i$. The other two parameters specify player $i$ 's payoff if she invests:

$$
u_{i}(a, \theta)= \begin{cases}b_{i}-c_{i} & \text { if } a \geq 1-\theta \\ -c_{i} & \text { if } a<1-\theta\end{cases}
$$

That is, the project succeeds if the aggregate action reaches the critical level $1-\theta$. Here $\theta$ - the fundamental - measures how propitious the environment is for the success of the project. We follow the literature in that higher $\theta$ makes investment more attractive, as a lower aggregate action is sufficient for success. We normalize the utility from not investing to $0 .^{10}$

In many applications, the outcome of the project is not binary, as assumed in equation (3); rather, it can result in various shades of success or failure. We extend our results to such payoff functions in the online Appendix.

The population of players consists of $G$ groups, indexed by $g \in\{1, \ldots, G\}$, each of measure $m_{g}$, with $\sum_{g} m_{g}=m$. Within a given group $g$ the members share the same values of parameters $w_{g}, c_{g}$, and $b_{g}$. We normalize $\sum_{g} w_{g} m_{g}=1$, so that the aggregate action varies between 0 and 1 .

The players have private information about the critical level of investment, $1-\theta$. (All other model parameters are publicly observed.) The explicit modeling of this fundamental uncertainty will allow us to describe the strategic uncertainty that the players face.

Each player $i$ is endowed with a type, which comprises a pair of numbers $\left(x_{i}, g_{i}\right)$. The first number is a noisy private signal about the fundamental, $x_{i}=\theta+\sigma \eta_{i}$, where $\sigma \in(0,1]$ scales the random error $\eta_{i}$. The second number labels the group to which player $i$ belongs. The pair $\left(\eta_{i}, g_{i}\right)$ is a random variable that is i.i.d. across players, and independent of $\theta$. The marginal probability, $\operatorname{Pr}\left(g_{i}=g\right)$, equals the population share, $m_{g} / m$, of group $g$. The distribution function of the conditional error $\eta_{i} \mid\left(g_{i}=g\right)$ is denoted by $F_{g}(\cdot)$ with support $[-1 / 2,1 / 2]$. Thus, players from

\footnotetext{
${ }^{10}$ We can accommodate free-riding. If noninvestors benefit $\underline{b}_{i}$ and investors benefit $\bar{b}_{i}$ from the success of the project, then the function $u_{i}$ is interpreted as the difference between the payoff for investing and not investing, with $b_{i}=\bar{b}_{i}-\underline{b}_{i}$.
} 
different groups draw errors from (possibly) different distributions $F_{g}$. The associated densities, $f_{g}(\cdot)$, are continuous. The players have no prior information about $\theta$; it is uniformly distributed on $[\underline{\theta}, \bar{\theta}]$, with $\underline{\theta}<-\sigma / 2$ and $\bar{\theta}>1+\sigma / 2$. $^{11}$ Thus, apart from neighborhoods of $\underline{\theta}$ and $\bar{\theta}$, type $(x, g)$ believes that $\theta$ is $x-\sigma \eta_{g}$ where $\eta_{g}$ is drawn from $F_{g}$. Vice versa, conditional on $\theta$, the signal $x$ of a player from group $g$ is distributed according to $F_{g}\left(\frac{x-\theta}{\sigma}\right)$.

\section{Equilibrium and Strategic Uncertainty}

In this section we characterize the unique equilibrium, at the limit where the players receive precise signals about the fundamental. We also explain how our model captures strategic uncertainty and, in Subsection IIIA, we state our main technical result: a characterization of the strategic beliefs.

PROPOSITION 1: For each $\sigma \in(0,1]$, the studied game has a unique Bayes-Nash equilibrium. In it, each player follows a threshold strategy:

$$
a_{i}(x, g)= \begin{cases}1 & \text { if } x \geq x_{g}^{*} \\ 0 & \text { if } x<x_{g}^{*}\end{cases}
$$

As $\sigma \rightarrow 0$, all thresholds $x_{g}^{*}$ converge to a common limit $\theta^{*}$, where

$$
\theta^{*}=\sum_{g=1}^{G} m_{g} \frac{w_{g}}{b_{g}} c_{g}
$$

In short, as the noise vanishes, the players coordinate on investing whenever $\theta>$ $\theta^{*}$ and on not investing whenever $\theta<\theta^{*}$. This result is not driven by the particular payoff function. It relies mainly on the usual global game properties of strategic complementarity and state monotonicity: $u_{g}$ (weakly) increases in $a$ and $\theta$. Indeed, in the online Appendix we generalize Proposition 1 to a class of nonbinary payoffs that vary with both the level of aggregate investment and the fundamental.

The structure of the unique equilibrium is depicted in Figure 1. The threshold signals $x_{g}^{*}$ form a cluster of the scale of the typical error, $\sigma$. When the realized $\theta$ falls within a neighborhood of the cluster, the players miscoordinate their actions due to the errors in their private signals. When $\theta$ is sufficiently to the right of the cluster then all players $i$ receive their signals $x_{i}$ above their thresholds $x_{g_{i}}^{*}$ and hence they coordinate on investing. When $\theta$ is sufficiently below the cluster, they coordinate on

\footnotetext{
${ }^{11}$ The uniform prior is not too restrictive because, as Frankel, Morris, and Pauzner (2003) show, any wellbehaved prior belief about $\theta$ becomes approximately uninformative when the private signals are sufficiently precise. Consequently, for small $\sigma$, our setup approximates one with a nonuniform prior. Therefore, we can also capture situations in which the planner's intervention is a reaction to a worsened prospect for the fundamental, as long as she does not have private information, and the private information of economic agents is precise compared to the public information.
} 


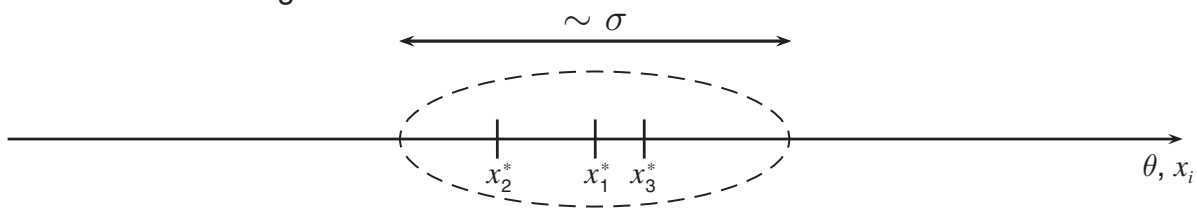

FIGURE 1

Note: An example of the equilibrium structure with three groups.

not investing. As the noise disappears, the cluster collapses to a point and there is no miscoordination.

The equilibrium is fully determined by the indifference conditions, $E\left[u_{g}(a, \theta) \mid\left(x_{g}^{*}, g\right)\right]=0$, of the players who receive their group's threshold signal $x_{g}^{*}{ }^{12}$ To clarify how these expectations are formed, we now discuss the notion of strategic beliefs.

Conditional on the realization of $\theta$, the measure of group $g$ players with signals above $x_{g}^{*}$, and therefore investing, is $m_{g}\left(1-F_{g}\left(\left(x_{g}^{*}-\theta\right) / \sigma\right)\right)$. Letting $\mathbf{x}^{*}$ denote the vector of threshold signals, the aggregate action is the following function of $\theta$ :

$$
\hat{a}\left(\theta, \mathbf{x}^{*}\right)=\sum_{g} w_{g} m_{g}\left(1-F_{g}\left(\frac{x_{g}^{*}-\theta}{\sigma}\right)\right) .
$$

The strategic belief of the threshold type of group $g$ is the cumulative distribution function $A_{g}(a)$ of the conditional random variable $\hat{a}\left(\theta, \mathbf{x}^{*}\right) \mid\left(x_{g}^{*}, g\right)$ : the probability that she assigns to the aggregate action being less than $a$.

The strategic beliefs depend, apart from the error distributions, only on the relative positions of the thresholds, defined as $\Delta_{g}=\left(x_{g}^{*}-x_{1}^{*}\right) / \sigma$. To see this, recall that type $\left(x_{g}^{*}, g\right)$ believes that $\theta=x_{g}^{*}-\sigma \eta_{g}$ with $\eta_{g} \sim F_{g}$ and hence we can write:

$$
A_{g}(a, \Delta)=\operatorname{Pr}\left(\sum_{h} w_{h} m_{h}\left(1-F_{h}\left(\Delta_{h}-\Delta_{g}+\eta_{g}\right)\right)<a\right),
$$

where $\Delta$ denotes the vector of $\Delta_{g}$.

When the errors in the observations of $\theta$ are small, each player knows that $\theta$ is close to her signal, so fundamental uncertainty is negligible. Yet strategic uncertainty is large even for precise signals. For any value of $\sigma$, the threshold types do not know how many of the players have observed their signals above or below their respective thresholds. As a result, they are uncertain about the aggregate action irrespective of the level of noise. Moreover, the strategic beliefs of the threshold types

\footnotetext{
${ }^{12}$ The beliefs of the nonthreshold types will not be needed in the analysis. If the threshold types satisfy the indifference conditions, then - as the incentive to invest is nondecreasing in the signal-all other types prefer their action prescribed by the monotone strategy profile.
} 


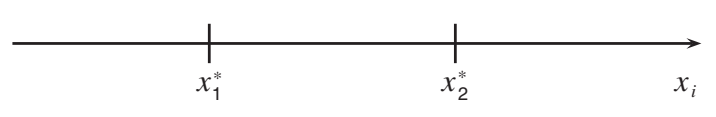

FIGURE 2

Note: The threshold types of groups 1 and 2 are pessimistic and optimistic, respectively, about the aggregate action.

may differ substantially across the groups: the relative differences in the thresholds, which determine these beliefs, do not vanish in the limit. As $\sigma \rightarrow 0$, the ratios $\left(x_{g}^{*}(\sigma)-x_{1}^{*}(\sigma)\right) / \sigma$ converge to some $\Delta_{g}^{*}$ for each group. Note that $\Delta_{1}=0$ by definition. The remaining $G$ variables, $\theta^{*}$ and $\Delta_{2}^{*}, \ldots, \Delta_{G}^{*}$, are the unique solution of the system of $G$ limit indifference conditions

$$
\int_{0}^{1} u_{g}\left(a, \theta^{*}\right) d A_{g}\left(a, \Delta^{*}\right)=0, \quad \text { for all } g .
$$

Direct analysis of these equations would be difficult because the strategic beliefs in equation (7) are complicated functions of the thresholds' relative positions as well as of the error distributions. Instead, we solve for $\theta^{*}$ indirectly, avoiding the full determination of strategic beliefs, using the result of the following section.

\section{A. The Belief Constraint}

Our equilibrium characterization is based on a generalization of the belief constraint discussed in the introduction. Recall the normalization $\sum_{g} w_{g} m_{g}=1$.

LEMMA 1 (The Belief Constraint): The (weighted) average strategic belief is the uniform belief on $[0,1]$ :

$$
\sum_{g=1}^{G} w_{g} m_{g} A_{g}(a, \Delta)=a .
$$

We prove Lemma 1 in the Appendix.

The robustness of this tight interdependence between the strategic beliefs is remarkable: it holds independently of the threshold positions, of the error distributions, and even of the payoffs. Furthermore, it is not a limit result; it holds for any $\sigma>0$.

The intuition behind the belief constraint can be partially gleaned from a simple example with two groups. Fix $\sigma>0$ and assume that the threshold types of groups 1 and 2 are ordered as in Figure $2 ; x_{1}^{*}<x_{2}^{*}$. Consider a player from group 1, who has received the threshold signal $x_{1}^{*}$. She is pessimistic about the investment from group 2 , as she expects others to receive signals near her own signal, $x_{1}^{*}$, and hence below $x_{2}^{*}$. Conversely, the threshold type of group 2 will be optimistic about investment from group 1. The optimism of one group is compensated by the pessimism of the other. Moving the thresholds closer or farther apart will have the opposite effect on the two strategic beliefs, keeping their average constant. The belief constraint states that such a relationship holds for any number of groups. 
Beyond providing a shortcut to calculate the equilibrium, the belief constraint also leads to an important economic insight: it shows that we can treat optimism about the aggregate action as a virtual resource, which is available in a fixed amount and is distributed among the groups. If, as a consequence of a policy intervention, the strategic belief of one group becomes more optimistic, then, to comply with the constraint, the other groups' strategic beliefs must become more pessimistic on average. Our policy analysis below builds on this intuition. It is based on a construction in which the policy shifts strategic optimism between the threshold types of different groups.

Empowered by the belief constraint, the equilibrium characterization becomes immediate. Let $p_{g}=\operatorname{Pr}\left(a>1-\theta^{*} \mid\left(x_{g}^{*}, g\right)\right)=1-A_{g}\left(1-\theta^{*}\right)$ denote the probability that the critical type of group $g$ assigns to the success of the project (we omit the second argument of $A_{g}$ for simplicity). The belief constraint has an important implication for the average of these beliefs:

$$
\sum_{g} m_{g} w_{g} p_{g}=\sum_{g} m_{g} w_{g}\left(1-A_{g}\left(1-\theta^{*}\right)\right)=1-\left(1-\theta^{*}\right)=\theta^{*}
$$

because the (weighted) average of $A_{g}(a)$ is the uniform distribution. Additionally, each threshold type satisfies the indifference condition

$$
b_{g} p_{g}-c_{g}=0 \text {. }
$$

The system of linear equations (9) and (10) has a unique solution with $\theta^{*}$ as specified in equation (5).

\section{The Planner's Problem}

The specific problem faced by an external agent who wishes to influence the outcome of the coordination game can be diverse, as both her objectives and the resources and instruments available to her differ across applications. In order to focus our discussion, we set the specific objective of achieving a given ex ante probability of successful coordination at minimal cost, using investment subsidies. We examine the limiting case of $\sigma \rightarrow 0$.

Consider a planner who publicly commits to pay subsidies $s_{g} \in\left[0, c_{g}\right]$ to all investing players. As a result, the effective cost of investing becomes $c_{g}-s_{g}$, and the critical state under the vector of subsidies $\mathbf{S}=\left(s_{g}\right)_{g}$ becomes

$$
\theta^{* *}(\mathbf{s})=\sum_{g=1}^{G} m_{g} \frac{w_{g}}{b_{g}}\left(c_{g}-s_{g}\right) .
$$

We define the planner's problem as

$$
\min _{\mathbf{S}} \sum_{g=1}^{G} m_{g} s_{g}
$$




$$
\text { subject to } \theta^{* *}(\mathbf{s})=\theta^{*} \text {, and } s_{g} \in\left[0, c_{g}\right] \text { for all } g .
$$

The motivation behind the optimization problem (11) is as follows. The planner incurs expenditures $\sum_{g} m_{g} s_{g}$ when the realized $\theta$ exceeds $\theta^{*}$ and zero expenditures for states below $\theta^{*} .^{13}$ Thus, of all schemes $\mathbf{s}$ that implement $\theta^{*}$, the solution of (11) minimizes the planner's expenditures in all contingencies (apart from a vanishing neighborhood of the critical state).

Assume that the planner has chosen threshold $\theta^{*} \in\left[0, \theta^{* *}(\mathbf{0})\right]$ so that there exists a feasible scheme implementing it. The following proposition characterizes the optimal subsidy scheme. Relabeling the groups in decreasing order of the ratios $w_{g} / b_{g}$ (ruling out the nongeneric case of ties), we have

PROPOSITION 2: The planner targets subsidies on the groups with the largest $w_{g} / b_{g}$ ratio: There exists a unique $g^{*}$ such that the optimal scheme $\mathbf{s}^{*}$ fully subsidizes groups $g<g^{*}$, does not subsidize groups $g>g^{*}$, and partially subsidizes group $g^{*}: s_{g}^{*}=c_{g}$ for $g<g^{*}, s_{g}^{*}=0$ for $g>g^{*}$, and $s_{g^{*}}^{*} \in\left[0, c_{g^{*}}\right]$.

The information the planner needs to choose her optimal subsidy targets is low, as she need not even know the probability distribution of the player's private information.

Thanks to the characterization result in Proposition 1, the planner's problem reduces to the straightforward linear optimization problem (11). The proof of Proposition 2 below highlights the role of the belief constraint, focusing on the optimal management of strategic uncertainty. The optimal policy intervention shifts strategic uncertainty from groups that are susceptible to it to the less susceptible groups. As the proof does not rely on the equilibrium characterization, it can be extended to a large payoff class that does not exhibit a closed-form solution. The generalization in the online Appendix confirms the basic insight: the optimal subsidy targets are agents who have a high impact on the aggregate action but whose incentives are not too sensitive to it.

\section{PROOF OF PROPOSITION 2:}

We prove the claim by contradiction. Consider a scheme $\mathbf{s}$ implementing threshold $\theta^{*}$ that differs in its structure from $\mathbf{s}^{*}$ specified in the proposition. We find an alternative scheme, $\mathbf{s}^{\prime}$, that implements $\theta^{*}$ with lower expenditure than $\mathbf{s}$. The scheme $\mathbf{s}^{\prime}$ is constructed as follows. As $\mathbf{S}$ violates the proposition, there exist two groups $e<f$ such that the two constraints $s_{e} \leq c_{e}$ and $s_{f} \geq 0$ are not binding. Thus, we can construct $\mathbf{s}^{\prime}$ by increasing the subsidy for group $e, s_{e}^{\prime}\left(s_{e}, c_{e}\right]$, decreasing the subsidy for group $f, s_{f}^{\prime} \in\left[0, s_{f}\right.$, and leaving all the other subsidies unaltered; $s_{g}^{\prime}=s_{g}$ for $g \notin\{e, f\}$.

Let us examine the strategic beliefs of the threshold types. The modified subsidies induce a change in the relative positions of the equilibrium thresholds and hence a change in the probabilities that the respective threshold types assign to success (from $p_{g}$ to $p_{g}^{\prime}$ ).

The threshold types satisfy indifference conditions under both schemes,

$$
b_{g} p_{g}-c_{g}+s_{g}=0=b_{g} p_{g}^{\prime}-c_{g}+s_{g}^{\prime} .
$$

\footnotetext{
${ }^{13}$ To simplify the discussion, we assume that an indifferent player invests if she is certain that the project will succeed and does not invest if she is certain that it will fail.
} 
As the subsidies for groups $g \notin\{e, f\}$ are not modified, only the success probabilities of groups $e$ and $f$ change. Putting this together with (the consequence of) the belief constraint (9), we obtain that the weighted change in success probability $m_{f} w_{f}\left(p_{f}^{\prime}-p_{f}\right)$ of group $f$ is exactly offset by the change $m_{e} w_{e}\left(p_{e}^{\prime}-p_{e}\right)$ for group $e$. Using the indifference conditions (10) again, we can relate these changes in success probabilities to the changes in subsidies:

$$
\frac{w_{e}}{b_{e}} m_{e}\left(s_{e}^{\prime}-s_{e}\right)=\frac{w_{f}}{b_{f}} m_{h} f\left(s_{f}-s_{f}^{\prime}\right)
$$

As $w_{e} / b_{e}>w_{f} / b_{f}$, the increase of expenditures on group $e, m_{e}\left(s_{e}^{\prime}-s_{e}\right)$, is smaller than the decrease of expenditures on group $f, m_{f}\left(s_{f}-s_{f}^{\prime}\right)$, as needed for the contradiction.

By equation (5), the identity and the received subsidy of the marginal group — the pair $\left(g^{*}, s_{g}^{*}\right)$-is the unique solution of

$$
m_{g^{*}} \frac{w_{g^{*}}}{b_{g^{*}}}\left(c_{g^{*}}-s_{g^{*}}\right)+\sum_{g=g^{*}+1}^{G} m_{g} \frac{w_{g}}{b_{g}} c_{g}=\theta^{*} .
$$

The resulting expenditure function, $\sum_{g} m_{g} s_{g}^{*}\left(\theta^{*}\right)$, specifies the possibility frontier of the planner. Her remaining task is to choose a pair $\left(\theta^{*}, \mathbf{s}^{*}\right)$ from this frontier, depending on how she trades off an increase in the ex ante probability of successful coordination against subsidy expenditures. We do not model this trade-off here. Instead, we briefly discuss a salient alternative to the planner's problem presented above.

As in the shopping mall example in the introduction, planners may be able to cross-subsidize investments across different groups of investors. To illustrate this, we consider a planner who subsidizes and taxes investment under a balanced budget in order to maximize the probability of success. Formally, her problem becomes

$$
\begin{aligned}
& \min _{\mathbf{s}} \sum_{g} m_{g} \frac{w_{g}}{b_{g}}\left(c_{g}-s_{g}\right) \\
& \text { subject to } \sum_{g} m_{g} s_{g}=0 \text { and } s_{g} \in\left[c_{g}-b_{g}, c_{g}\right] \quad \text { for all } g .
\end{aligned}
$$

It is straightforward to verify that the planner will fully subsidize groups with the highest $w_{g} / b_{g}$ ratios, fully tax groups with the lowest ratios, while she will partially change the investment incentives of a middle group, either taxing or subsidizing it.

\section{Discussion}

Proposition 2 singles out the optimal targets for intervention. More generally interpreted, the proposition answers the question in the title of the paper. It identifies the players who matter most in the coordination process, in the sense that the equilibrium outcome is more sensitive to a variation of their investment costs than to those of the other players. Let us discuss what makes players influential. 
In accordance with common intuition, high weight $w_{g}$-that is, high direct impact on the aggregate action-makes one influential. This suggests that economic sectors whose performance has large spillovers on the rest of the economy are natural targets of stimulus. In the recent public debate, the argument for bailouts of the automobile and the banking industries was that keeping these running is essential for keeping the whole economy active. ${ }^{14}$

Our result suggests that the above intuition should be refined: another feature that makes a group influential is low $b_{g}$; that is, its relative insensitivity to aggregate economic activity. This suggests that the public debate should focus on an amended question. Are the spillovers of, say, the automobile sector imposed on the whole economy large relative to the sensitivity of the automobile sector to the performance of the whole economy? As the automobile sector is procyclical, and hence quite sensitive to the state of the economy, the answer to the question is less than obvious.

The high influence of insensitive players has external validity beyond the global game framework. Their influence is high because of the indirect rather than the direct effect of their subsidies. The size of the direct effect of the subsidy $s_{g}$ on the investment incentive $b_{g} p_{g}-c_{g}+s_{g}$ is independent of $b_{g}$. But, if the targeted players are the most insensitive ones - the ones with the lowest $b_{g}$ - then the unsubsidized players are, by construction, sensitive to the others' actions. These- untargeted-sensitive players are keen to invest in response to an increased investing activity of the targeted players. Thus, our targeting rule maximizes the indirect effect of the subsidies.

We also identify factors that do not affect a group's influence, among them the investment costs $c_{g}$. It is tempting to target small subsidies on players with low $c_{g} / b_{g}$ ratio; that is, on those for whom the investing is nearly dominant. Such targeting is not generally optimal, however, as the untargeted agents may happen to be the ones with low sensitivity to the aggregate action and hence may be unimpressed by the increased investment activity of the subsidized agents.

Our model predicts that neither the group sizes $m_{g}$ are relevant for the targeting. Naturally, one dollar subsidy for each member of a large group has a larger impact on the economy than one dollar subsidy promised to members of a small group. But the first scheme implies higher expenditures for the planner than the latter, and these two effects cancel out perfectly. Thus, within our analysis, there is no reason to subsidize a large industry at the expense of smaller ones. ${ }^{15}$

Finally, our model rejects the tempting conjecture that players with superior information about the fundamental exhibit larger influence on the coordination outcome. Both the equilibrium characterization and the optimal subsidy targeting are independent of the assumed error distributions, and a relative increase in the precision of a group's signals does not affect the influence of the group.

\footnotetext{
${ }^{14}$ Robert F. Brunner argues in the New York Times ("U.S. Not Always Averse to Nationalization, Despite its Free-Market Image," October 13, 2008) why the financial as opposed to some other distressed sector needs to be bailed out: "the spillover effects from the crisis in the financial system are so great, pulling down the rest of the economy in a way that no other industry can, so that the potential cost of not doing something like this is immense."

${ }^{15}$ We do not examine the influence of large individual players. Corsetti et al. (2004) explain why large players, such as the iconic George Soros, have large influence over the coordination of many small players. These effects are not present in our model, in which all players are individually small.
} 


\section{Selected Applications}

There are a host of practical problems in which economic agents may coordinate on an inefficient equilibrium. Our main contribution, a good understanding of how the heterogeneity of agents affects the best way to tackle these problems, is often particularly relevant. In this section we provide a few representative examples in which our insights are useful.

\section{A. Financial Fragility}

The Basel Committee on Banking Supervision has long recognized that a onesize-fits-all approach to banking regulation is suboptimal. The Basel II (2004) accords introduced "microprudential" regulation, allowing for a customized (credit) risk evaluation of each financial intermediary, largely based on the composition of its outlays. A lesson reinforced in the recent financial crisis is that, especially to avoid systemic crises, regulation must also aim to keep solvent banks from failing due to liquidity problems, caused by the structure of their liabilities and the behavior of their creditors (refusing to roll over, withdrawing early, refusing to lend, etc.); see, for example, Morris and Shin (2008, 2012) and Vives (2011).

Our technique can combine the heterogeneity of the liability structure now emphasized by practitioners (witness the Basel III (2011) accords) with the commonly used theoretical models of liquidity crises. Below we provide two examples that, though highly stylized, may help to inform debates about financial regulation.

Targeted Deposit Freeze.-The recent paper of Ennis and Keister (2009) discusses the practice of imperfect deposit freezes. As they illustrate with the 2001-2002 Argentinian crisis, deposit freezes are often not absolute: e.g., courts may allow depositors with basic liquidity needs to withdraw. Discriminatory deposit freezes create a complex welfare dilemma: by allowing some withdrawals, the government increases the likelihood of a run in the first place, but once the run occurs the benevolent planner prefers to respect the liquidity needs of depositors. Our technique allows us to track how a discriminatory withdrawal policy affects the probability of a run, thus informing policy discussion. Consider the following, highly stylized, variation of our main model.

There are $G$ groups of depositors of total measure 1, who have all deposited $\$ 1$ at date 0 in a bank. At date 1, they may attempt to withdraw their money. A group $g$ agent who attempts to withdraw succeeds with probability $q_{g} \in[0,1]$, chosen ex ante by the regulator. The groups differ in their need for liquidity: the utility gain from successful withdrawal is $c_{g}>0$.

Those who have not withdrawn, either because they have not tried or they have failed, are paid off at date 2 . The date 2 payoff is $R>1$ if the bank survives and 0 if it fails. The depositors vary in their outside investment opportunities and thus they have heterogeneous discount factors $\rho_{g}$. We let $b_{g}=R \rho_{g}>c_{g}$ be the (date 1) present value payoff from keeping the deposit in the bank, if the bank survives. Finally, the bank survives if the volume of deposits kept in the bank after date 1 is at least $1-\theta$. Using the belief constraint, we show in the online Appendix that the resulting critical state is $\theta^{*}=\sum_{g} m_{g} q_{g} c_{g} / b_{g}$. 
Let us turn to the regulator's problem. She first decides what probability of bank failure she is willing to tolerate, effectively choosing $\theta^{*}$. If $\theta>\theta^{*}$, there will be no run. If $\theta<\theta^{*}$, all depositors will attempt to withdraw. The regulator, wishing to maximize the welfare gain from these withdrawals, solves

$$
\max _{\left(q_{g}\right) g} \sum_{g} m_{g} q_{g} c_{g}, \text { subject to } \sum_{g} m_{g} q_{g} \frac{c_{g}}{b_{g}}=\theta^{*} .
$$

The solution confirms the intuition from our baseline planner's problem in Section IV. Irrespective of their liquidity needs, $c_{g}$, the regulator should allow withdrawal, $q_{g}=1$, for the groups who are least impatient, with the highest $b_{g}$, while it should ban withdrawals, $q_{g}=0$, for the most impatient groups, with the lowest $b_{g}$. As in the baseline case, the policy maximizes the indirect effects of the partial deposit freeze. By construction, the agents who are allowed to withdraw are sensitive to the aggregate action, and hence their incentive to roll over the deposit greatly increases with the withdrawal ban targeted on their peers. A naive policy that, in a hope to stop the avalanche of withdrawals, bans withdrawals of agents with high need for liquidity (high $c_{g}$ ) does not optimize the indirect effects of the regulation and leads to welfare losses.

Discriminatory Deposit Insurance.-Though full deposit insurance would eliminate bank runs, in practice there are limits to the amount that can be credibly insured. ${ }^{16}$ We modify our model to investigate a protocol that optimizes over a set of discriminatory deposit insurance schemes. Depositors from group $g$ can withdraw their deposits $c_{g}$ early and receive payoff $c_{g}$ or withdraw late. Late payment is 0 if the bank fails and $b_{g}=c_{g}\left(1+r_{g}\right)$ if the bank does not fail. The bank fails if the total withdrawal exceeds $\theta$. Notice that the setup is the special case of our baseline model with weights $w_{g}=c_{g}$. A planner provides investors with insurance against the bank's failure. She commits to pay $c_{g} i_{g} \in\left[0, c_{g}\right]$ to all continuing depositors from group $g$ if the bank fails, which effectively changes cost parameters to $c_{g}-c_{g} i_{g}$ and benefit parameters to $b_{g}-c_{g} i_{g}$.

The critical state under the policy becomes

$$
\theta^{*}=\sum_{g} m_{g} w_{g} \frac{c_{g}-c_{g} i_{g}}{b_{g}-c_{g} i_{g}}=\sum_{g} m_{g} c_{g} \frac{1-i_{g}}{1+r_{g}-i_{g}} .
$$

Consider a planner committed to a marginal expenditure $e$ deciding which group to target. If she targets group $g$, providing insurance $c_{g} i_{g}=e / m_{g}$ to each member, then the critical state changes by approximately

$$
\left.m_{g} c_{g} \frac{d}{d i_{g}}\left(\frac{1-i_{g}}{1+r_{g}-i_{g}}\right)\right|_{i_{g}=0} \frac{e}{m_{g} c_{g}}=-\frac{r_{g}}{\left(1+r_{g}\right)^{2}} e .
$$

\footnotetext{
${ }^{16}$ Even with the recent rises due to the crisis the stated limits are restrictive: in Canada C $\$ 100,000$; in the United States $\$ 250,000$; in Euroland it varies between $€ 50,000$ and $€ 100,000$; in the United Kingdom $£ 50,000$; and in Mexico UDI400,000 $\approx \operatorname{Mex} \$ 88,000$. In a systemic crisis it is unlikely that the insurers would pay out even these amounts.
} 
For a realistic range of interest rate, $r_{g} \in[0,1]$, the right-hand side decreases in $r_{g}$. Consequently, the planner, who minimizes $\theta^{*}$, prefers to insure the investors who are promised a high interest rate, irrespective of the size of their deposit.

Targeting the insurance on a group with high $r_{g} /\left(1+r_{g}\right)^{2}$ is intuitive. Notice that

$$
\frac{r_{g}}{\left(1+r_{g}\right)^{2}}=\frac{1}{1+r_{g}}\left(1-\frac{1}{1+r_{g}}\right)=\frac{w_{g}}{b_{g}}\left(1-p_{g}\right),
$$

where $p_{g}=1 /\left(1+r_{g}\right)$ again denotes the critical success probability that makes the agent indifferent. We see the familiar effect that the optimal targets are the influential and insensitive players, the ones with high $w_{g} / b_{g}$. The additional criterion, targeting agents with high $1-p_{g}$, is also intuitive: threshold types with high $1-p_{g}$ assign high probability to receiving the payout, and thus are responsive to its promise.

\section{B. Network Goods}

Ryan and Tucker (2012) analyze the role of individual heterogeneity in the diffusion of a network technology. Using a detailed dataset on the adoption of a new videoconferencing technology in a large investment bank, they estimate a structural model of technology adoption and use. They find that employees have significant heterogeneity in both adoption costs and network benefits. Based on their estimates, they evaluate counterfactual policies of exogenously seeding a group to adopt at the start. The difference across different targets is substantial: the best seeding performs nearly two-thirds better than the worst one.

Our model can be easily adapted to find the optimal seeding policy. Let $\overline{\mathbf{m}}=\left(\bar{m}_{1}, \bar{m}_{2}, \ldots, \bar{m}_{G}\right)$ be the measure of people who are "forced" to adopt in each group. Furthermore, assume that the planner internalizes proportion $\alpha$ of the investment costs of the employees who are compelled to adopt. Then for a given budget $w$ the planner maximizing the adoption probability solves

$$
\min _{\overline{\mathbf{m}}} \sum_{g} w_{g}\left(m_{g}-\bar{m}_{g}\right) \frac{c_{g}}{b_{g}} \quad \text { such that } \sum \bar{m}_{g} \alpha c_{g}=w
$$

leading to the familiar result that the seeded employees should be the ones with the highest $w_{g} / b_{g}$.

Financial markets are equally exposed to network externalities that lead to coordination problems, which can be alleviated by targeted policies. Cantillon and Yin $(2009,2011)$ document the fight between two financial exchanges, London International Financial Futures and Options Exchange and Deutsche Terminbörse, to attract traders in an important future on a long-term German government bond (the Bund). As traders benefit from liquidity, ceteris paribus they prefer to trade at the more popular market, making this a coordination problem. The authors demonstrate that there is significant diversity among traders' preference for liquidity, though they differ on other, "horizontal," characteristics affecting their choice of market as well. This heterogeneity was utilized by the competing platforms, who targeted various groups of traders with preferential treatments. We can complement these empirical findings by pointing out that, unlike their actual choice of market, 
the influence of traders is not affected by horizontal characteristics. Rather, the platforms should target their subsidies to those traders who substantially contribute to liquidity, but at the same time have only a weak preference for it.

\section{Conclusion}

Our model suggests that a planner aiming to coordinate agents on high economic activity should subsidize the subset of agents who are relatively insensitive to the aggregate action. Compared to uniform subsidies, targeted subsidies can be high per recipient, and thus can have large leverage, not only on the targeted agents, but also on the untargeted ones, who, by the choice of the targeting rule, are sensitive to the actions of others.

We formalize this intuition in a global game model with heterogeneous payoffs. The analysis focuses on the strategic beliefs, captured as the beliefs about aggregate action held by threshold types. We show that these beliefs are interrelated by a simple constraint: their average across all groups is the uniform belief.

The model emphasizes that subsidies cannot eliminate strategic uncertainty from the coordination process. There always remains a critical range of fundamentals within which players suffer from large strategic uncertainty. Though the critical range may be small, it is pivotal for the coordination outcome. The planner, unable to eliminate strategic uncertainty, should focus on its optimal management. This is reflected by our optimal subsidy targeting, which shifts strategic pessimism to those groups that are the least sensitive to the behavior of others, thus minimizing the adverse effects of strategic uncertainty.

Let us revisit the development example of the introduction. The empirical analysis of government interventions that proved successful in giving the "big push" for development has identified two distinct families of policy. One of them is the "picking winners" approach, which allocates subsidies based on "economic criteria," like growth potential (see, for example, Rodrik 1995). The other type of intervention is horizontal; it is not openly favoring a specific sector or industry, but still has differential effects on different parts of the economy. A good example of this is an undervalued local currency, a factor that Rodrik (2008) identifies as key to successful development. Our results can contribute toward a better understanding of both types of policies.

Our subsidy targeting model can be directly applied to the "picking winners" scenario. It suggests that basing the subsidy mostly on the growth potential or the likelihood of localized success is a suboptimal policy. When there are important externalities, a government should make use of indirect incentives, where the subsidization of one industry mobilizes investment in another one. Counter-cyclical industries are appropriate subsidy targets as these are the ones who benefit relatively little from an overall increase in economic activity. Thus, their subsidization has a relatively large indirect impact on the untargeted, procyclical industries.

Our model can be used to evaluate and compare horizontal policies as well. In the real exchange rate example of Rodrik (2008), the policy clearly favors tradables versus nontradables. He argues that such a policy must have been successful, because tradables suffer disproportionately from market failures and institutional weaknesses. Our model suggests that it is unclear that tradables are the best target 
for (implicit) subsidies as, while it is more difficult/costly for them to get on the bandwagon, they also have higher potential benefits from it.

Let us conclude with a thought on coordinating on higher economic activity in a developed country. The American Recovery and Reinvestment Act of 2009 authorized the spending of $\$ 787$ billion to jump-start the US economy. A package of such magnitude has obviously had a visible impact, and it has created/saved many jobs (in the first quarter of 2011 more than 560,000 jobs were "Act funded"). ${ }^{17}$ Nonetheless, it is noteworthy that the disbursement of grants, contracts, loans, tax credits, and entitlements has been devolved to 28 different federal agencies. What are the chances that, amid local political pressures favoring "fair" sharing, these agencies coordinated on prioritizing those who matter most in the coordination problem of recovery?

\section{APPENDIX}

\section{The Proof of the Belief Constraint}

This Appendix derives our core technical observation that allows for the analysis of coordination in a heterogeneous population.

Before proving the belief constraint, we discuss its special case for a homogeneous population. Assume that the signal errors are independently, identically distributed across all players, who all use the same cutoff $x^{*}$. Then the aggregate action is simply $\hat{a}\left(\theta, x^{*}\right)=\operatorname{Pr}\left(x_{i}>x^{*} \mid \theta\right)$. The following property, driving characterization results in most global game applications, is our belief constraint applied to such a symmetric situation:

LAPLACIAN PROPERTY (Morris and Shin 2003): In the symmetric setup, the player who receives the threshold signal $x^{*}$ believes that the aggregate action is distributed uniformly on $[0,1]$ :

$$
\hat{a}\left(\theta, x^{*}\right) \mid x^{*} \sim U[0,1] .
$$

We will rephrase and prove the Laplacian Property below in the context of the proof of the belief constraint.

The property has an intuitive explanation. We paraphrase Morris and Shin (2003): The threshold type, $x^{*}$, constitutes a boundary in between the investing and noninvesting types. She is uncertain about the realized proportions of types above and below the boundary. These proportions are determined by the rank of her signal within the realized population of players' signals. The only information the threshold

\footnotetext{
${ }^{17}$ Source: www.recovery.gov.
} 
type receives is her own private signal, which is entirely uninformative about the rank of her signal and consequently about the aggregate action.

Our belief constraint establishes that the Laplacian property holds on average across the threshold types of the groups. Its proof consists of finding a virtual homogeneous problem in which the strategic belief (partially) characterizes the beliefs in the original heterogeneous problem. ${ }^{18}$

PROOF OF LEMMA 1 (The Belief Constraint):

Let us define the mapping $\delta\left(x_{i}, g_{i}\right)=x_{i}-x_{g_{i}}^{*}$ that reduces the two-dimensional type $\left(x_{i}, g_{i}\right)$ to a one-dimensional virtual signal $\tilde{x}_{i}=\delta\left(x_{i}, g_{i}\right)$. The strategy

$$
a_{i}\left(x_{i}, g_{i}\right)= \begin{cases}1 & \text { if } x_{i} \geq x_{g_{i}}^{*} \\ 0 & \text { if } x_{i}<x_{g_{i}}^{*}\end{cases}
$$

depends on the type $\left(x_{i}, g_{i}\right)$ only via the virtual signal $\tilde{x}_{i}: a_{i}\left(x_{i}, g_{i}\right) \equiv \tilde{a}_{i}\left(\delta\left(x_{i}, g_{i}\right)\right)$, where

$$
\tilde{a}_{i}\left(\tilde{x}_{i}\right)= \begin{cases}1 & \text { if } \tilde{x}_{i} \geq \tilde{x}^{*} \\ 0 & \text { if } \tilde{x}_{i}<\tilde{x}^{*}\end{cases}
$$

with the virtual threshold $\tilde{x}^{*}$ set to 0 .

Recall that $\hat{a}\left(\theta, \mathbf{x}^{*}\right)$ denotes the aggregate action and consider the conditional random variable $\hat{a}\left(\theta, \mathbf{x}^{*}\right) \mid\left(\tilde{x}_{i}=\tilde{x}^{*}\right)$. It can be interpreted as a belief about the aggregate action of a player who knows that her virtual signal is $\tilde{x}_{i}=\tilde{x}^{*}$, but "forgot" her original type $\left(x_{i}, g_{i}\right)$.

The expression for the aggregate action (6), restated here for convenience,

$$
\hat{a}\left(\theta, \mathbf{x}^{*}\right)=\sum_{g} w_{g} m_{g}\left(1-F_{g}\left(\frac{x_{g}^{*}-\theta}{\sigma}\right)\right),
$$

can be written as

$$
\hat{a}\left(\theta, \mathbf{x}^{*}\right)=\operatorname{Pr}^{\prime}\left(\left\{(x, g): x \geq x_{g}^{*}\right\} \mid \theta\right)
$$

where $\operatorname{Pr}^{\prime}$ denotes the modified probability measure that assigns to the error-group pair $(\eta, g)$ probability density $w_{g} m_{g} f_{g}(\eta)$ instead of the original measure Pr that assigns to $(\eta, g)$ density $f_{g}(\eta) m_{g} / m$. Using the definition of the virtual signal, the expression for the aggregate action can be rewritten as

$$
\hat{a}\left(\theta, \mathbf{x}^{*}\right)=\operatorname{Pr}^{\prime}\left(\tilde{x}_{i} \geq \tilde{x}^{*} \mid \theta\right)
$$

\footnotetext{
${ }^{18}$ A similar proof strategy is used by Kováč and Steiner (2009) in a different context. They study a dynamic global game and reduce the complex strategic belief in the dynamic environment to a simple, well-understood belief in a static game.
} 
All players use the same strategy with identical virtual threshold signal $\tilde{x}^{*}$ and all are identical at the ex ante stage. In this symmetric environment, no information about the aggregate action can be derived from the event that a player has received the threshold virtual signal:

LEMMA 2 (Laplacian Property (Morris and Shin 2003)): The aggregate action is, conditional on the threshold virtual signal, uniformly distributed on $[0,1]$ :

$$
\operatorname{Pr}^{\prime}\left(\hat{a}\left(\theta, \mathbf{x}^{*}\right)<a \mid \tilde{x}_{i}=\tilde{x}^{*}\right)=a \text {. }
$$

See the proof below.

Finally, we relate the belief of the virtual threshold type $\tilde{x}^{*}$ to the beliefs of the original threshold types $\left(x_{g}^{*}, g\right)$. Due to the translation invariance of the information structure, $g_{i} \mid \tilde{x}_{i}$ is independent of $\tilde{x}_{i}$ and so the conditional probability equals the prior probability; $\operatorname{Pr}^{\prime}\left(g_{i}=g \mid \tilde{x}_{i}\right)=w_{g} m_{g}$. Thus, the virtual signal is entirely uninformative about player $i$ 's original group identity:

LEMMA 3:

$$
\operatorname{Pr}^{\prime}\left(\left(x_{i}, g_{i}\right)=\left(x_{g}^{*}, g\right) \mid \tilde{x}_{i}=\tilde{x}^{*}\right)=w_{g} m_{g}
$$

for all groups $g$.

See the proof below.

As a result, the strategic belief of the virtual threshold type is the following compound lottery:

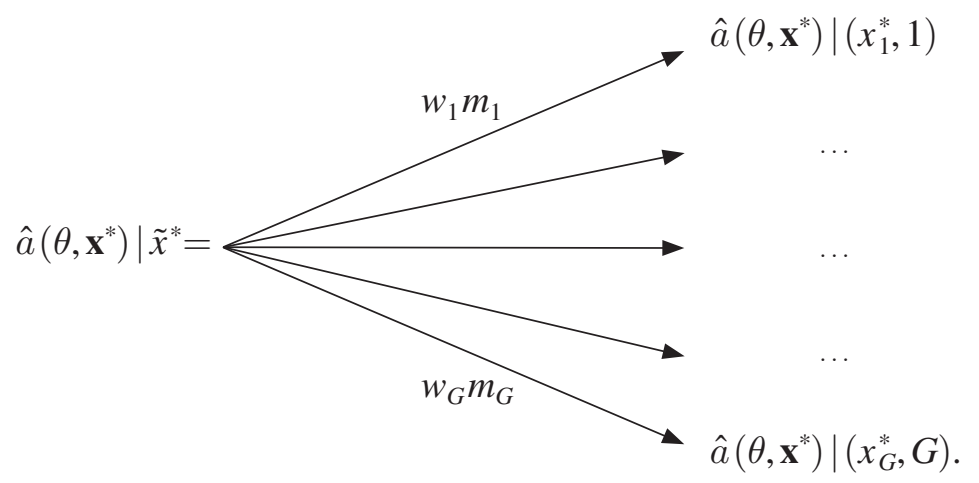

Hence, since the left-hand side is distributed uniformly by Lemma 2, and recalling that the cumulative distribution function of $\hat{a}\left(\theta, \mathbf{x}^{*}\right) \mid\left(x_{g}^{*}, g\right)$ is $A_{g}(a)$, we obtain $a=\sum_{g} w_{g} m_{g} A_{g}(a)$. 
PROOF OF LEMMA 2 (based on Morris and Shin 2003):

The virtual error defined as $\tilde{\eta}_{i}=\left(\tilde{x}_{i}-\theta\right) / \sigma$ is a compound lottery

$$
\left(\eta_{i 1}-\frac{x_{1}^{*}}{\sigma}, \ldots, \eta_{i G}-\frac{x_{G}^{*}}{\sigma} ; w_{1} m_{1}, \ldots, w_{G} m_{G}\right)
$$

where $\eta_{i g}$ are random variables drawn from distributions $F_{g}$, independent of $\theta$ and across $i$. Therefore $\tilde{\eta}_{i}$ is i.i.d. across players, and also independent of $\theta$. (The bias of the virtual signal, $E\left[\tilde{\eta}_{i}\right] \neq 0$, is not relevant for the argument.) Let $\tilde{F}$ denote the distribution function of $\tilde{\eta}_{i}$. As $F_{g}$ is strictly increasing for each $g, \tilde{F}$ is strictly increasing as well, and thus its inverse function is well defined. Below we show that $\hat{a}$ $(\theta) \mid\left(\tilde{x}_{i}=\tilde{x}^{*}\right)$ is indeed uniformly distributed.

$$
\begin{aligned}
& \operatorname{Pr}^{\prime}\left(\hat{a}(\theta)<z \mid \tilde{x}_{i}=\tilde{x}^{*}\right)= \\
& \operatorname{Pr}^{\prime}\left(\operatorname{Pr}^{\prime}\left(\tilde{x}_{j}>\tilde{x}^{*} \mid \theta\right)<z \mid \tilde{x}_{i}=\tilde{x}^{*}\right)= \\
& \operatorname{Pr}^{\prime}\left(\operatorname{Pr}^{\prime}\left(\tilde{\eta}_{j}>\frac{\tilde{x}^{*}-\theta}{\sigma}\right)<z \mid \tilde{x}_{i}=\tilde{x}^{*}\right)= \\
& \operatorname{Pr}^{\prime}\left(1-\tilde{F}\left(\frac{\tilde{x}^{*}-\theta}{\sigma}\right)<z \mid \tilde{x}_{i}=\tilde{x}^{*}\right)= \\
& \operatorname{Pr}^{\prime}\left(1-\tilde{F}\left(\tilde{\eta}_{i}\right)<z\right)= \\
& \operatorname{Pr}^{\prime}\left(\tilde{\eta} i>\tilde{F}^{-1}(1-z)\right)= \\
& 1-\tilde{F}(\tilde{F}(1-z))=z .
\end{aligned}
$$

\section{PROOF OF LEMMA 3:}

We fix player $i$ and omit her index. We write capital letter for a random variable, and small letter for its realization. To distinguish it from the number of groups $G$, we denote the random variable describing player $i$ 's group by $G^{\prime}$.

Let $\left(\Theta, X, G^{\prime}\right)$ be a random variable describing the fundamental and the type of player $i$. The modified probability density of $\left(\Theta, X, G^{\prime}\right)$ is

$$
p_{\Theta, X, G^{\prime}}^{\prime}(\theta, x, g)=\frac{1}{\bar{\theta}-\underline{\theta}} f_{g}\left(\frac{x-\theta}{\sigma}\right) \frac{1}{\sigma} w_{g} m_{g},
$$

where $f_{g}(\cdot)$ is the density of $\eta_{i} \mid\left(g_{i}=g\right)$.

The marginal probability density of $\left(X, G^{\prime}\right)$ is

$$
p_{X, G^{\prime}}^{\prime}(x, g)=\int_{x-\sigma / 2}^{x+\sigma / 2} \frac{1}{\bar{\theta}-\underline{\theta}} f_{g}\left(\frac{x-\theta}{\sigma}\right) w_{g} m_{g} \frac{d \theta}{\sigma}=\frac{w_{g} m_{g}}{\bar{\theta}-\underline{\theta}}
$$


for all $x \in[\underline{\theta}+\sigma / 2, \bar{\theta}-\sigma / 2]$. The marginal probability density of the virtual signal $\tilde{x}=x-x_{g}^{*}$ is

$$
p_{\tilde{X}}^{\prime}(\tilde{x})=\sum_{g} p_{X, G^{\prime}}^{\prime}\left(\tilde{x}+x_{g}^{*}, g\right)=\sum_{g} \frac{w_{g} m_{g}}{\bar{\theta}-\underline{\theta}}=\frac{1}{\bar{\theta}-\underline{\theta}},
$$

if

$$
\tilde{x}+x_{g}^{*} \in[\underline{\theta}+\sigma / 2, \bar{\theta}-\sigma / 2] \text { for each } g .
$$

Finally, if $\tilde{x}$ satisfies (A1) then

$$
\operatorname{Pr}^{\prime}\left(G^{\prime}=g \mid \tilde{X}=\tilde{x}\right)=\frac{p_{X, G^{\prime}}^{\prime}\left(\tilde{x}+x_{g}^{*}, g\right)}{p_{\tilde{X}}^{\prime}(\tilde{x})}=w_{g} m_{g} .
$$

Notice that $\tilde{x}=\tilde{x}^{*}=0$ satisfies (A1) because thresholds $x_{g}^{*}$ outside of $[\underline{\theta}+\sigma / 2$, $\bar{\theta}-\sigma / 2]$ violate the indifference condition, as a player receiving such a signal knows that $\theta$ lies in a dominance region.

The remaining proofs are relegated to the online Appendix.

\section{REFERENCES}

Basel Committee on Banking Supervision. 2004. Basel II: International Convergence of Capital Measurement and Capital Standards: A Revised Framework. Basel, Switzerland: Bank for International Settlements.

Basel Committee on Banking Supervision. 2011. Basel III: A Global Regulatory Framework for More Resilient Banks and Banking Systems_Revised Version June 2011. Basel, Switzerland: Bank for International Settlements.

Bebchuk, Lucian A., and Itay Goldstein. 2011. "Self-Fulfilling Credit Market Freezes." Review of Financial Studies 24 (11): 3519-55.

Bernstein, Shai, and Eyal Winter. 2012. "Contracting with Heterogeneous Externalities." American Economic Journal: Microeconomics 4 (2): 50-76.

Camerer, Colin F. 2003. Behavioral Game Theory: Experiments in Strategic Interaction. Princeton, NJ: Princeton University Press.

Cantillon, Estelle, and Pai-Ling Yin. 2009. “Asymmetric Network Effects.” The Networks, Electronic Commerce, and Telecommunications Institute Working Paper 08-42.

Cantillon, Estelle, and Pai-Ling Yin. 2011. "Competition between Exchanges: Lessons from the Battle of the Bund." http://www.econ2.uni-bonn.de/downloads/bund-battle-10-july-2011.pdf (accessed September 26, 2012).

Carlsson, Hans, and Eric van Damme. 1993. "Global Games and Equilibrium Selection.” Econometrica 61 (5): 989-1018.

Corsetti, Giancarlo, Bernardo Guimarães, and Nouriel Roubini. 2006. "International Lending of Last Resort and Moral Hazard: A Model of IMF's Catalytic Finance." Journal of Monetary Economics 53 (3): 441-71.

Corsetti, Giancarlo, Amil Dasgupta, Stephen Morris, and Hyun Song Shin. 2004. "Does One Soros Make a Difference? A Theory of Currency Crises with Large and Small Traders." Review of Economic Studies 71 (1): 87-113.

Diamond, Douglas W., and Philip H. Dybvig. 1983. "Bank Runs, Deposit Insurance, and Liquidity." Journal of Political Economy 91 (3): 401-19.

Edmond, Chris. 2011. "Information Manipulation, Coordination, and Regime Change." National Bureau of Economic Research Working Paper 17395.

Ennis, Huberto M., and Todd Keister. 2009. "Bank Runs and Institutions: The Perils of Intervention." American Economic Review 99 (4): 1588-1607.

Farrell, Joseph, and Garth Saloner. 1985. "Standardization, Compatability, and Innovation." RAND Journal of Economics 16 (1): 70-83. 
Frankel, David M., Stephen Morris, and Ady Pauzner. 2003. "Equilibrium Selection in Global Games with Strategic Complementarities.” Journal of Economic Theory 108 (1): 1-44.

Goldstein, Itay, and Ady Pauzner. 2004. "Contagion of Self-Fulfilling Financial Crises Due to Diversification of Investment Portfolios.” Journal of Economic Theory 119 (1): 151-83.

Goldstein, Itay, and Ady Pauzner. 2005. "Demand-Deposit Contracts and the Probability of Bank Runs.” Journal of Finance 60 (3): 1293-1327.

Gould, Eric D., B. Peter Pashigian, and Canice J. Prendergast. 2005. "Contracts, Externalities, and Incentives in Shopping Malls." Review of Economics and Statistics 87 (3): 411-22.

Guimarães, Bernardo, and Stephen Morris. 2007. "Risk and Wealth in a Model of Self-Fulfilling Currency Attacks." Journal of Monetary Economics 54 (8): 2205-30.

Harsanyi, John C., and Reinhard Selten. 1988. A General Theory of Equilibrium Selection in Games. Cambridge, MA: MIT Press.

Izmalkov, Sergei, and Muhamet Yildiz. 2010. "Investor Sentiments." American Economic Journal: Microeconomics 2 (1): 21-38.

Kandori, Michihiro, George J. Mailath, and Rafael Rob. 1993. "Learning, Mutation, and Long-Run Equilibria in Games." Econometrica 61 (1): 29-56.

Katz, Michael L., and Carl Shapiro. 1986. "Technology Adoption in the Presence of Network Externalities." Journal of Political Economy 94 (4): 822-41.

Kiyotaki, Nobuhiro, and Randall Wright. 1989. "On Money as a Medium of Exchange." Journal of Political Economy 97 (4): 927-54.

Kováč, Eugen, and Jakub Steiner. 2009. "Reversibility in Dynamic Coordination Problems.” University of Edinburgh School of Economics Discussion Paper 183.

König, Philipp J., and Tijmen R. Daniëls. 2011. "Liquidity and Solvency in a Model of Emerging Market Crises." Unpublished.

Mathevet, Laurent. 2011. "Beliefs and Rationalizability in Games with Complementarities.” Unpublished. https://webspace.utexas.edu/lm26875/www/documents/Beliefs_Rationalizability.pdf.

Matsui, Akihiko, and Kiminori Matsuyama. 1995. "An Approach to Equilibrium Selection.” Journal of Economic Theory 65 (2): 415-34.

Morris, Stephen, and Hyun Song Shin. 1998. "Unique Equilibrium in a Model of Self-Fulfilling Currency Attacks." American Economic Review 88 (3): 587-97.

Morris, Stephen, and Hyun Song Shin. 2003. "Global Games: Theory and Applications.” In Advances in Economics and Econometrics: Eighth World Congress, edited by M. Dewatripont, M. Hansen, and S. Turnovsky, 56-114. Cambridge, UK: Cambridge University Press.

Morris, Stephen, and Hyun Song Shin. 2004a. "Coordination Risk and the Price of Debt." European Economic Review 48 (1): 133-53.

Morris, Stephen, and Hyun Song Shin. 2004b. "Liquidity Black Holes.” Review of Finance 8 (1): 1-18.

Morris, Stephen, and Hyun Song Shin. 2006. "Catalytic Finance: When Does It Work?" Journal of International Economics 70 (1): 161-77.

Morris, Stephen, and Hyun Song Shin. 2008. "Financial Regulation in a System Context." Brookings Papers on Economic Activity 2008: 229-61.

Morris, Stephen, and Hyun Song Shin. 2012. "Contagious Adverse Selection.” American Economic Journal: Macroeconomics 4 (1): 1-21.

Obstfeld, Maurice. 1996. "Models of Currency Crises with Self-Fulfilling Features." European Economic Review 40 (3-5): 1037-47.

Pashigian, B. Peter, and Eric D. Gould. 1998. "Internalizing Externalities: The Pricing of Space in Shopping Malls.” Journal of Law and Economics 41 (1): 115-42.

Ray, Debraj. 2000. "What's New in Development Economics?" American Economist 44 (2): 3-16.

Rodrik, Dani. 1995. "Getting Interventions Right: How South Korea and Taiwan Grew Rich." Economic Policy: A European Forum 20: 53-97.

Rodrik, Dani. 2008. "The Real Exchange Rate and Economic Growth." Brookings Papers on Economic Activity 2008: 365-412.

Ryan, Stephen, and Catherine Tucker. 2012. "Heterogeneity and the Dynamics of Technology Adoption." Quantitative Marketing and Economics 10 (1): 63-109.

Sah, Raaj K. 1991. "Social Osmosis and Patterns of Crime." Journal of Political Economy 99 (6): 1272-95.

Segal, Ilya. 1999. “Contracting with Externalities.” Quarterly Journal of Economics 114 (2): 337-88.

Segal, Ilya. 2003. "Coordination and Discrimination in Contracting with Externalities: Divide and Conquer?" Journal of Economic Theory 113 (2): 147-81.

Vives, Xavier. 2011. "Strategic Complementarity, Fragility, and Regulation.” Instituto de Estudios Superiores de la Empresa (IESE) Discussion Paper D/928-E. 